\title{
Effect of Hyperglycemia Treatment on Complications Rate after Pediatric Cardiac Surgery: a randomized clinical trail
}

\author{
Bahman Naghipour ${ }^{1}$, Mahdieh Bagerpour ${ }^{1}$, Kamran Shadvar ${ }^{1}$, Samad EJ Golzari ${ }^{1}$, and \\ Gholamreza Faridaalaee ${ }^{1}$
}

${ }^{1}$ Tabriz University of Medical Sciences

May 6, 2021

\begin{abstract}
Objective(s): The goal of this study was to elucidate harmful complications of intraoperative hyperglycemia following children cardiac surgery and benefits of insulin administration for accurate blood sugar controlling. Design: Randomized clinical trial Setting: operating room of shahid madani hospital Patients: 50 patients who were children under 12 years old undergoing cardiac surgery using Cardiopulmonary Bypass (CPB). Interventions: Intraoperative insulin infusion was administered intravenously targeting at blood sugar levels of 110-140mg/dL Measurements and Main Results: Blood sugar and ABG were measured every 30 min during operation. The means of hospitalization and extubation time were more in the placebo than study group $(\mathrm{P}=0.03)$ and $(\mathrm{P}=0.005)$, respectively. However, the mean of hospitalization in the ICU ward was not significantly between groups. Conclusions: Hyperglycemia prevalence was lower in our study than mentioned studies in which the patients were either given insulin or not. Hyperglycemia duration has relation with long hospitalization time in ICU and hospital. These findings suggest the positive effect of accurate blood sugar control on reducing complication and hospitalization time in children undergoing cardiac surgery.
\end{abstract}

\section{Hosted file}

main document.pdf available at https://authorea.com/users/412299/articles/521029-effectof-hyperglycemia-treatment-on-complications-rate-after-pediatric-cardiac-surgery-arandomized-clinical-trail 


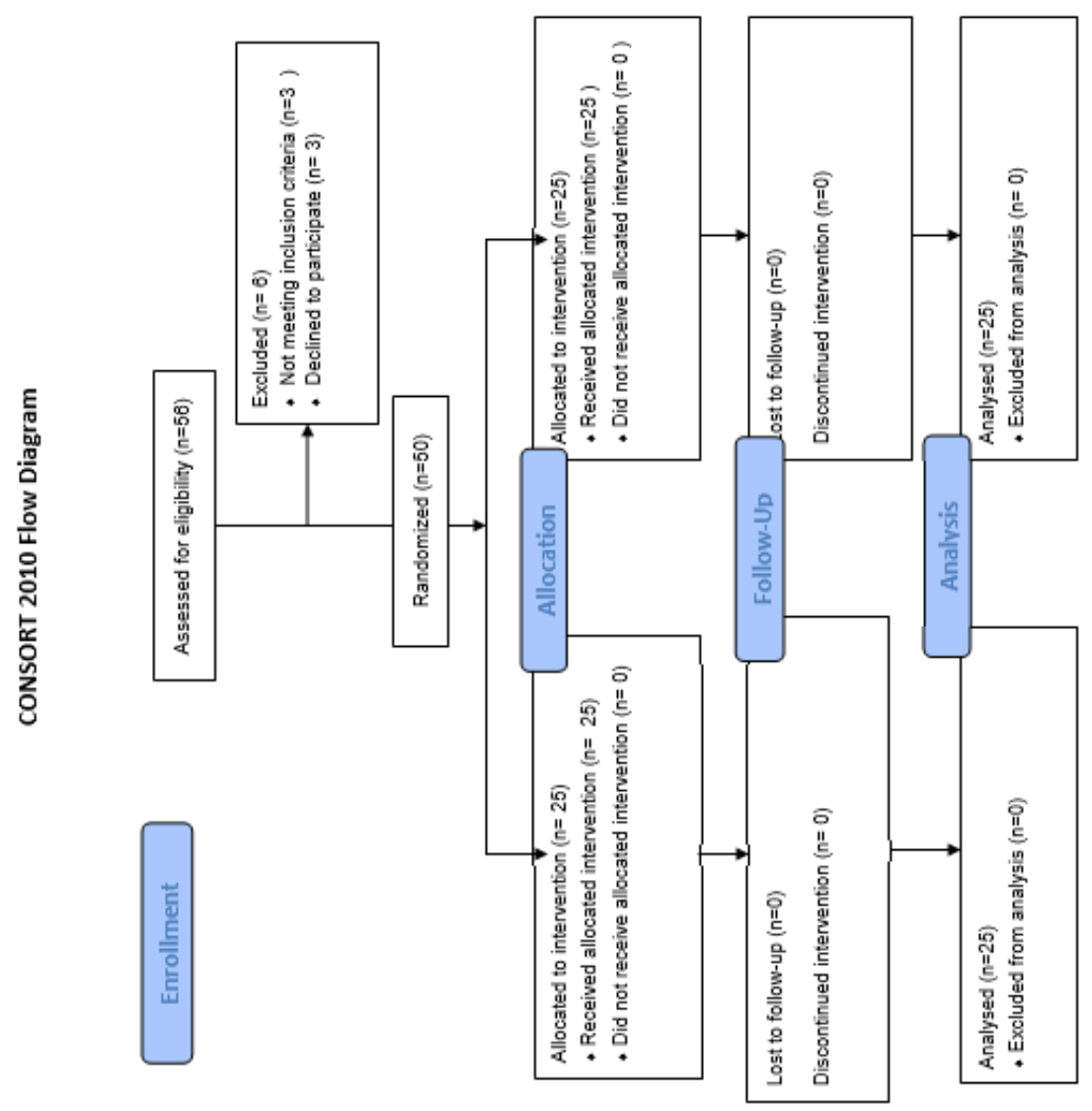

\section{Hosted file}

Table 1.pdf available at https://authorea.com/users/412299/articles/521029-effect-ofhyperglycemia-treatment-on-complications-rate-after-pediatric-cardiac-surgery-arandomized-clinical-trail

\section{Hosted file}

Table 2.pdf available at https://authorea.com/users/412299/articles/521029-effect-ofhyperglycemia-treatment-on-complications-rate-after-pediatric-cardiac-surgery-arandomized-clinical-trail

\section{Hosted file}

Table 3.pdf available at https://authorea.com/users/412299/articles/521029-effect-ofhyperglycemia-treatment-on-complications-rate-after-pediatric-cardiac-surgery-arandomized-clinical-trail

\section{Hosted file}

Table 4.pdf available at https://authorea.com/users/412299/articles/521029-effect-ofhyperglycemia-treatment-on-complications-rate-after-pediatric-cardiac-surgery-arandomized-clinical-trail

\section{Hosted file}


Table 5.pdf available at https://authorea.com/users/412299/articles/521029-effect-ofhyperglycemia-treatment-on-complications-rate-after-pediatric-cardiac-surgery-arandomized-clinical-trail 